\title{
KCT : a MATLAB toolbox for motion control of KUKA robot manipulators
}

\author{
Francesco Chinello, Stefano Scheggi, Fabio Morbidi, Domenico Prattichizzo
}

\begin{abstract}
The Kuka Control Toolbox (KCT) is a collection of MATLAB functions for motion control of KUKA robot manipulators, developed to offer an intuitive and high-level programming interface to the user. The toolbox, which is compatible with all 6 DOF small and low payload KUKA robots that use the Eth.RSIXML, runs on a remote computer connected with the KUKA controller via TCP/IP. KCT includes more than 30 functions, spanning operations such as forward and inverse kinematics computation, point-to-point joint and Cartesian control, trajectory generation, graphical display, 3-D animation and diagnostics. The flexibility, ease of use and reliability of the toolbox is demonstrated through two applicative examples.
\end{abstract}

\section{INTRODUCTION}

MATLAB [1] is a powerful and widely used commercial software environment for numerical computations, statistic analysis and graphical presentations available for a large number of platforms. Specific toolboxes (i.e., collection of dedicated MATLAB functions) have been developed in the past few years for support in research and education, in almost every branch of engineering, such as, e.g., telecommunications, electronics, aerospace, mechanics and control. As far as robotics is concerned, several toolboxes have been presented in the last decade for the modeling of robot systems [2]-[7]. These simulation tools have been inspired by various applicative scenarios, such as robotic vision [5], [6] and space robotics [3], and have addressed different targets ranging from industrial [4] to academiceducational [2], [5]-[7]. A more challenging problem is to design MATLAB toolkits, offering versatile and highlevel programming environments, for motion control of real robots. Some work has been done in this field for one of the first industrial robots, the Puma 560 manipulator [8], [9]: however this robot is known to have some intrinsic software limitations, especially in real-time applications, which have been overcome by more recent manipulators.

In this paper we will focus on the manipulators produced by KUKA [10], one of the world's leading manufacturers of industrial robots. KUKA manipulators are designed to cover a large variety of applications in industrial settings, such as, e.g., assembly, material handling, dispensing, palletizing and welding tasks. A specific C-like programming language, called KRL (KUKA Robot Language), has been developed by KUKA for robot motion control. This language is simple and allows comfortable programming. However,

The authors are with the Department of Information Engineering, University of Siena, 53100 Siena, Italy. List of e-mails:

\{chinello, scheggi,morbidi,prattichizzo\}@dii.unisi.it it is not suited for critical real-time remote control applications, it does not support graphical interfaces and advanced calculus (such as, matrix operations, optimization and filtering tasks), and it does not allow an easy integration of external modules and hardware (such as, e.g., cameras or embedded devices using common protocols: USB, Firewire, PCI, etc.). A possible way to overcome these drawbacks is to build a MATLAB abstraction layer upon the KRL. A first step torwards this direction has been recently taken by a MATLAB toolbox called Kuka-KRL-tbx [11]. The authors use a serial interface to connect the KUKA Robot Controller (KRC) with a remote computer including MATLAB. A KRL interpreter, running on the $\mathrm{KRC}$, realizes a bi-directional communication between the robot and the remote computer and it is responsible for the identification and execution of all intructions that are transmitted via the serial interface. $K u k a-K R L-t b x$ offers a homogeneous environment from the early design to the operation phase and an easy integration of external hardware components. In addition, it preserves the security standards guaranteed by the KRL (workspace supervision, check of the final position switches of every robot axis, etc.).

However, Kuka-KRL-tbx suffers from some limitations:

- The MATLAB commands of the toolbox are one-toone with the KRL functions: this lack of abstraction may hinder the user from designing advanced control applications.

- The serial interface may represent a limit in real-time control applications.

- The toolbox does not include specific routines for graphical display.

This paper presents a new MATLAB toolbox, called KUKA Control Toolbox (KCT), for motion control of KUKA robot manipulators. The toolbox, designed both for academic/educational and industrial purposes, includes a broad set of functions divided into 6 categories, spanning operations such as, forward and inverse kinematics computation, point-to-point joint and Cartesian control, trajectory generation, graphical display, 3-D animation and diagnostics.

KCT shares with Kuka-KRL-tbx the same advantages and improves it in several directions:

- The functions of KCT are not a MATLAB counterpart of the corresponding KRL commands. This makes the toolbox extremely flexible and versatile.

- KCT runs on a remote computer connected with the KRC via TCP/IP. A multi-thread server runs on the KRC and communicates via Eth.RSIXML (Ethernet 


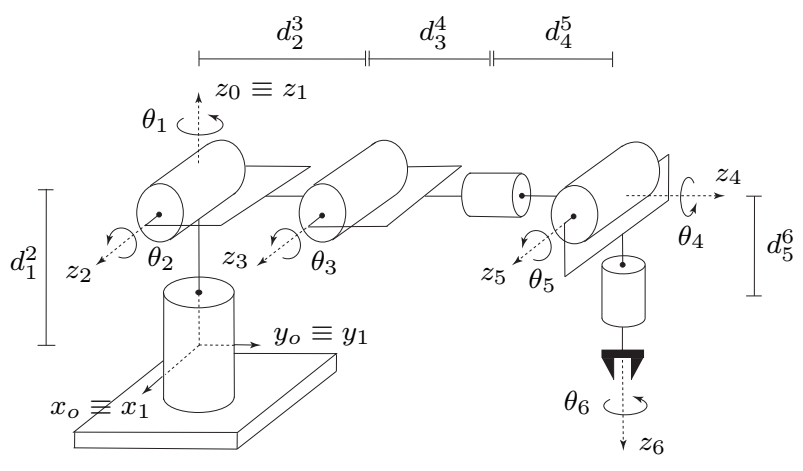

Fig. 1. Reference robot: 6 DOF elbow manipulator with spherical wrist.

Robot Sensor Interface XML) with a client managing the information exchange with the manipulator. This communication scheme guarantees high transmission rates, thus enabling real-time control applications.

- KCT has several dedicated functions for graphics and animation, and includes a graphical user interface.

- The toolbox and the relative documentation can be freely downloaded from the web page: http://sirslab.dii.unisi.it/vision/kct

$\mathrm{KCT}$ is fully compatible with all small and low payload 6 DOF KUKA robot manipulators which run the 5.4, 5.5 or $7.0 \mathrm{KSS}$ (Kuka System Software): the controllers KR C2, KR C2 ed05 and KR C3 (equipped with a real-time $10 / 100$ card) are currently supported by the toolbox. KCT can be easily integrated with other MATLAB toolboxes and it has been successfully tested on multiple platforms, including Windows, Mac and Linux.

The rest of the paper is organized as follows: Sect. II provides a comprehensive overview of the functions of KCT. Two examples are reported in Sect. III to show the flexibility of the toolbox in real scenarios. In Sect. IV, conclusions are drawn and future research directions are highlighted.

\section{OVERVIEW OF THE TOOLBOX}

In this section the functions of KCT will be briefly described. The 6 DOF robot manipulator shown in Fig. 1 will be considered as a reference along the paper: we will use the vector $\mathbf{q}=\left[\theta_{1}, \theta_{2}, \ldots, \theta_{6}\right]^{T}$ to denote the collection of the joint angles of the manipulator and $\mathbf{d}_{k-1}^{k} \in \mathbb{R}^{3}, k \in$ $\{1,2, \ldots, 6\}$, to indicate the displacement between the center of the $(k-1)$-th and $k$-th joint of the robot (note that $\left.\mathbf{d}_{0}^{1} \equiv \mathbf{0}\right)$. The homogeneous matrix $\mathbf{H}_{0}^{6} \in \mathrm{SE}(3)$ relates the coordinates of a $3-\mathrm{D}$ point written in the base reference frame $\left\langle x_{0}, y_{0}, z_{0}\right\rangle$, with the coordinates of the same point written in the end-effector frame $\left\langle x_{6}, y_{6}, z_{6}\right\rangle$.

In the interest of clarity, the commands of KCT have been subdivided into 6 categories, according to the task they perform (see Table II, next page). The KUKA robot models currently supported by KCT are listed in Table I: up to now, the toolbox has been successfully tested on the KR3, KR5sixxr850 and KR16 robots. Fig. 2 illustrates the communication scheme between $\mathrm{KCT}$ and the robot manipulator. It consists of three parts:

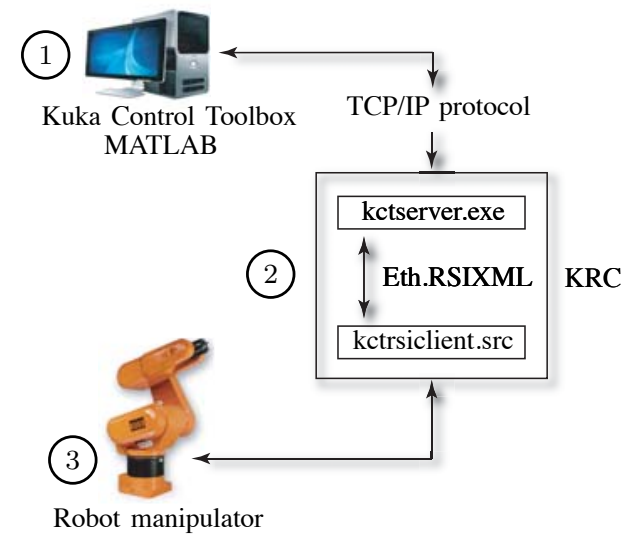

Fig. 2. Communication scheme between KCT and the manipulator.

1) A remote computer running KCT under MATLAB,

2) The KUKA Robot Controller (KRC),

3) The robot manipulator.

To establish a connection between the remote computer and the robot controller, KCT provides kctserver, a C++ multi-thread server running on the KRC. kctserver communicates via Eth.RSIXML (a KUKA software package for TCP/IP-robot interface) with kctrsiclient.src, a KRL client running on the $\mathrm{KRC}$ and managing the information exchange with the robot manipulator. The MATLAB functions of KCT communicate with kctserver using specific MEX-files (default option) or via the Instrument Control Toolbox. The server sends the robot's current state to the computer and the velocity commands to the manipulator via kctrsiclient.src, in a soft real-time loop of $15 \pm 1 \mathrm{~ms}$ (the communication has been temporized to $15 \mathrm{~ms}$ in order to have a suitable margin over the $12 \mathrm{~ms}$ physical time limit: the $\pm 1 \mathrm{~ms}$ uncertainty is currently due to the lack of a hard real-time support platform). kctrsiclient.src is also used to define a номе position (starting position) for the manipulator. Hardware and software contraints on robot's motion are established by Eth.RSIXML. The hardware constraints depend on manipulator's physics and cannot be modified by the user. On the contrary, the software constraints can be configured at the beginning of each working session via the functions kctrsiclient.src or kctsetbound (see Sect. II-A for more details).

In the following, all the angles will be in degrees and distances in millimeters.

\begin{tabular}{|c|c|c|c|c|}
\hline \multicolumn{5}{|c|}{ Small Robots (from 3 to $5 \mathrm{~kg}$ ) } \\
\hline KR3 & KR5sixxr650 & KR & sixxr850 & \\
\hline \multicolumn{5}{|c|}{ Low Payloads (from 6 to $16 \mathrm{~kg}$ ) } \\
\hline KR5arc & KR5arcHW & KR6-2 & KR6-2KS & \\
\hline KR16-2 & KR16-2S & KR16-2KS & KR16L6-2 & KR16L6-2KS \\
\hline
\end{tabular}

TABLE I

6 DOF KUKA ROBOTS CURRENTLY SUPPORTED BY KCT. 


\begin{tabular}{|c|c|}
\hline \multicolumn{2}{|l|}{ Initialization } \\
\hline kctrobot & Show the list of supported KUKA robots \\
\hline kctinit & Load the parameters of the selected robot \\
\hline kctsetbound & Set the workspace bounds \\
\hline kctgetbound & Visualize the workspace bounds \\
\hline \multicolumn{2}{|l|}{ Networking } \\
\hline kctclient & Initialize the client \\
\hline kctcloseclient & Terminate the client \\
\hline \multicolumn{2}{|l|}{ Kinematics } \\
\hline kctreadstate & Return the current configuration of the robot \\
\hline kctfkine & Compute the forward kinematics \\
\hline kctikine & Compute the inverse kinematics \\
\hline kctfkinerpy & Compute the forward kinematics (give the pose) \\
\hline kctikinerpy & Compute the inverse kinematics (from the pose) \\
\hline \multicolumn{2}{|l|}{ Motion control } \\
\hline kctsetjoint & Set the joint angles to a desired value \\
\hline kctsetxyz & Move the end-effector in a desired position \\
\hline kctmovejoint & Set the joint velocities to a desired value \\
\hline kctmovexyz & $\begin{array}{l}\text { Move the end-effector with a desired linear } \\
\text { and angular velocity }\end{array}$ \\
\hline kctdrivegui & GUI for robot motion control \\
\hline kctpathxyz & Generate a trajectory (operational space) \\
\hline kctpathjoint & Generate a trajectory (joint space) \\
\hline kcthome & Drive the robot back to the initial position \\
\hline kctstop & Stop the robot in the current position \\
\hline kctdemo & Demonstration of the toolbox \\
\hline \multicolumn{2}{|l|}{ Graphics } \\
\hline kctdisptraj & Plot the 3-D trajectory of the end-effector \\
\hline kctdispdyn & Plot the time history of the joint angles \\
\hline kctanimtraj & Create a 3-D animation of the robot \\
\hline \multicolumn{2}{|c|}{ Homogeneous transforms } \\
\hline kctrotox & Hom. transform for rotation about $x$-axis \\
\hline kctrotoy & Hom. transform for rotation about $y$-axis \\
\hline kctrotoz & Hom. transform for rotation about $z$-axis \\
\hline kcttran & Hom. transform for translation \\
\hline kctchframe & Change the reference frame \\
\hline
\end{tabular}

TABLE II

LIST OF KCT FUNCTIONS DIVIDED BY CATEGORY.

\section{A. Initialization}

The information relative to the KUKA robots supported by KCT is stored in the MATLAB file kctrobotdata.mat (see Table III) and can be accessed by typing,

> kctrobot( );

To initialize a particular robot model, it is sufficient to write kctinit ('KR3'), where the argument is a string containing the name of the robot selected (e.g., KR3, KR5sixxr650, KR5sixxr850, etc.) as specified in kctrobotdata.mat. The function kctsetbound(B) can be used to set the software bounds of the robot. The matrix,

$$
\mathbf{B}=\left[\begin{array}{cccccc}
X^{m} & X^{M} & Y^{m} & Y^{M} & Z^{m} & Z^{M} \\
\theta_{4}^{m} & \theta_{4}^{M} & \theta_{5}^{m} & \theta_{5}^{M} & \theta_{6}^{m} & \theta_{6}^{M}
\end{array}\right]
$$

contains the bounds on the position and orientation (limited to the joint angles $\theta_{4}, \theta_{5}$ and $\theta_{6}$ ) of the end-effector. Note that differently from kctrsiclient.src, kctsetbound enables a MATLAB warning message in the motion control

\begin{tabular}{|c|c|c|c|c|}
\hline 'name' & KR3 & KR5sixxr650 & KR5sixxr850 & $\ldots$ \\
\hline 'link1' [mm] & 350 & 335 & 335 & \\
'link2' [mm] & 100 & 75 & 75 & \\
'link3' [mm] & 265 & 270 & 365 & $\ldots$ \\
'link4' [mm] & 0 & 90 & 90 & \\
'link5' [mm] & 270 & 295 & 405 & \\
'link6' [mm] & 75 & 80 & 80 & \\
\hline
\end{tabular}

TABLE III

DATA STORED IN THE FILE KCTROBOTDATA.MAT.

functions (see Sect. II-D), when the workspace's bounds are violated. The bounds can be graphically visualized using the function kctgetbound.

\section{B. Networking}

After the initialization step, the TCP/IP communication between KCT and kctserver must be established. The main steps necessary to initialize the connection are the following:

1) Put the mode selector on $T 1$ (automatic execution) in the KUKA control panel (teach pendant).

2) Select kctrsiclient.src on KSS.

3) Start kctserver.

4) In the MATLAB workspace start the KCT/IP communication with kctserver by typing kctclient('193.155.1.0'), where 193.155.1.0 is the IP address of the KRC real-time network card.

5) Start kctrsiclient.src by pressing the run button on the KUKA control panel and keep pushing until the line ST_SKIPSENS is reached.

6) Check whether the communication is established. If it is not, return to step 2 .

To close the TCP/IP communication is sufficient to type kctcloseclient ().

\section{Kinematics}

The state of the manipulator is stored in a $2 \times 6$ matrix, called robotstate, containing the current position and roll-pitch-yaw orientation of the end-effector (first row), and the current joint angles (second row). This matrix can be accessed using the command: robotstate $=$ kctreadstate ( ). To compute the matrix $\mathbf{H}_{0}^{6}$ of the forward kinematics and the inverse kinematics solution expressed as a joint angles' vector $\mathbf{q}$, KCT provides the following two functions:

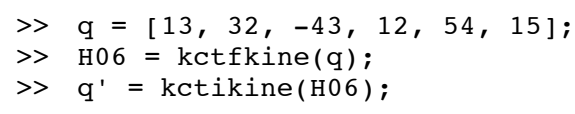

The command $\mathrm{p}=$ kctfkinerpy(q) is analogous to kctfkine, but returns the position and roll-pitch-yaw orientation of the end-effector of the robot, as a vector $\mathbf{p}=[X, Y, Z, \phi, \gamma, \psi]^{T}$. Finally, the function $\mathbf{q}=$ kctikinerpy (p) computes the inverse kinematics solution from the vector $\mathbf{p}$. 


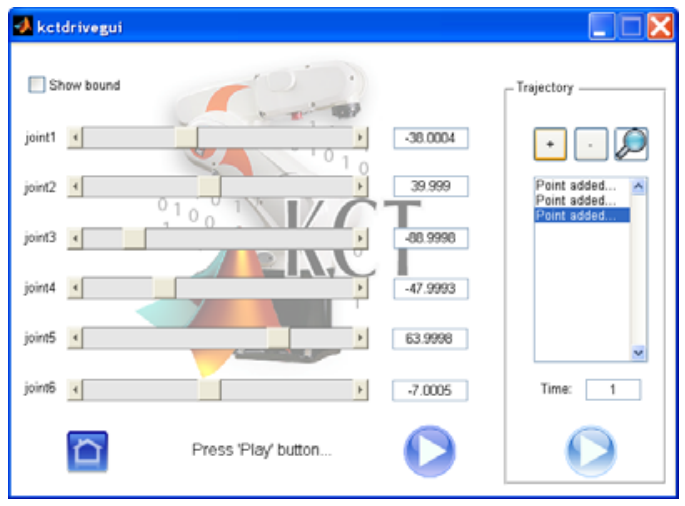

Fig. 3. The interface loaded by the function kctdrivegui.

\section{Motion control}

KCT provides several functions for point-to-point control and trajectory planning. The simplest task one could require, is to move the robot from an initial to a final configuration defined by robot's joint angles or by end-effector's poses. Let $\mathbf{q}_{f}=\left[\theta_{1}, \theta_{2}, \ldots, \theta_{6}\right]^{T}$ be the final desired joint configuration of the robot. The function,

$>$ qf $=[23,35,12,-21,54,60]$;

$>$ [robotinfo, warn] $=\operatorname{kctsetjoint}\left(\mathrm{qf}\right.$, 'poly' $\left.^{\prime}\right)$;

moves the robot from the current to the final configuration using either a polynomial approach [12, Sect. 5.5.2] or a proportional control, as specified by the second argument (poly or prop, respectively). The matrix robotinfo contains the time history of the joint angles and warn is a Boolean variable that is set to 1 when an error occurs during robot's motion. Let now $\mathbf{p}_{f}=[X, Y, Z, \phi, \gamma, \psi]^{T}$ be the final desired pose of the end-effector. The function,

$>$ pf $=[412,-2,350,20,12,15]$;

$>$ [robotinfo, warn] $=\operatorname{kctsetxyz}(\mathrm{pf})$;

moves to robot from the initial to the desired pose $\mathbf{p}_{f}$ using a proportional controller. Note that kctsetjoint and kctsetxyz are user-level routines relying on two lower level functions: kctmovejoint and kctmovexyz. When kctsetjoint is called, the KUKA controller computes the joint velocities necessary to accomplish the requested task using kctmovejoint(qdot). Similarly, when kctsetxyz is called, the linear and angular velocities of the endeffector necessary to achieve the goal are computed via kctmovexyz (pdot).

A graphical user interface, inspired by Robotics Toolbox's drivebot GUI [2], is loaded by kctdrivegui(), (see Fig. 3). The joint angles of the robot can be easily regulated here by acting on six sliders, and the corresponding motion of the robot is diplayed via a 3-D animation.

It is very frequent in the applications to deal with trajectories defined by a sequence of Cartesian frames or joint angles. Consider a sequence of $n$ points $\mathbf{p}_{i}=$ $\left[X_{i}, Y_{i}, Z_{i}, \phi_{i}, \gamma_{i}, \psi_{i}\right]^{T}, i \in\{1,2, \ldots, n\}$, stacked into the $n \times 6$ matrix $\mathbf{P}=\left[\mathbf{p}_{1}, \mathbf{p}_{2}, \ldots, \mathbf{p}_{n}\right]^{T}$. The following commands,

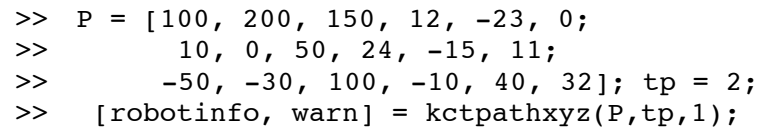

move the end-effector of the robot from point $\mathbf{p}_{1}$ to point $\mathbf{p}_{3}$ using a linear interpolation method: a time step tp of 2 seconds between two consecutive points is considered. The third argument of kctpathxyz is a Boolean variable enabling or disabling the visualization of the 3-D trajectory of the end-effector and the time history of the joint angles at the end of the task. The function kctpathjoint is analogous to kctpathxyz, the only difference being that the trajectory is defined in the joint space instead of the operational space. The first argument of kctpathjoint is a $n \times 6$ matrix $\mathbf{Q}$, whose rows are vectors of joint angles:

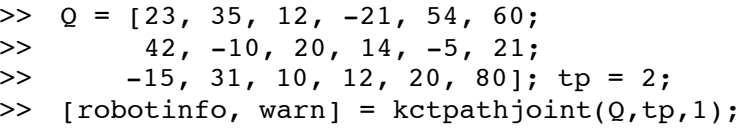

To immediately stop the robot in the current position, one should first terminate the execution of the motion control functions using ctrl-c, and then type kctstop(). Finally, to drive the robot back to the initial position, $\mathrm{KCT}$ provides the function kcthome( ).

\section{E. Graphics}

Three functions are available in KCT for graphical display. The function kctdisptraj(robotinfo) plots the 3-D trajectory of the end-effector, kctdispdyn(robotinfo) plots the time history of the robot joint angles and kctanimtraj(robotinfo) creates a 3-D animation of the robot executing the requested task. A suite of options (frame's and trajectory's color, frame's dimension, viewpoint, etc.) is available for the customization of the plot. (see the help of the single commands for more details).

\section{F. Homogeneous transforms}

KCT provides a set of transformation functions of frequent use in robotics. Let $\mathbf{d} \in \mathbb{R}^{3}$ be a translation vector and $\alpha$ an angle. The functions,

$>$ Htr $=\operatorname{kcttran}(\mathrm{d}) ; \mathrm{Hx}=\operatorname{kctrotox}(\mathrm{alpha}) ;$
$>$ Hy $=\operatorname{kctrotoy}(\mathrm{alpha}) ; \mathrm{Hz}=\operatorname{kctrotoz}(\mathrm{alpha}) ;$

provide the basic homogeneous transformations generating $\mathrm{SE}(3)$ for translation and rotation about the $x, y, z$-axes.

Let us now suppose that we wish to move the robot's end-effector with respect to an external reference frame $\left\langle x_{w}, y_{w}, z_{w}\right\rangle$ different from the base $\left\langle x_{0}, y_{0}, z_{0}\right\rangle$. This could be useful, for instance, in an eye-in-hand framework where robot's motion should be referred to the camera frame. Let $\mathbf{H}_{0}^{w}$ be the homogeneous matrix defining the rigid motion between $\left\langle x_{w}, y_{w}, z_{w}\right\rangle$ and $\left\langle x_{0}, y_{0}, z_{0}\right\rangle$. The function,

$>\mathrm{HOW}=\operatorname{kctrotoz}(\mathrm{alpha}) * \operatorname{kcttran}(\mathrm{d})$;

fixes $\left\langle x_{w}, y_{w}, z_{w}\right\rangle$ as new reference frame. All the operations specified by commands executed after kctchframe are referred to $\left\langle x_{w}, y_{w}, z_{w}\right\rangle$. 


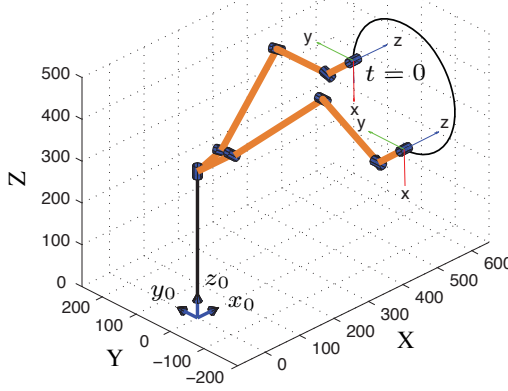

(a)
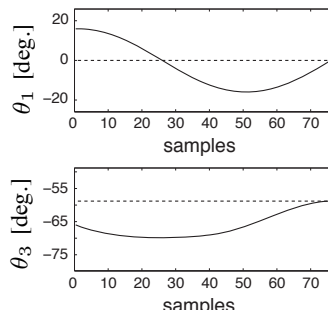

samples

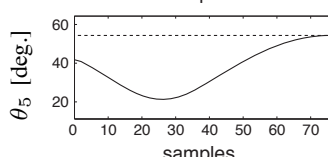

samples

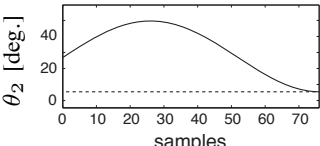

samples

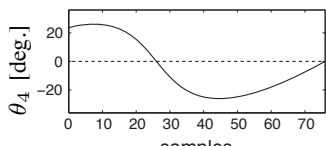

samples

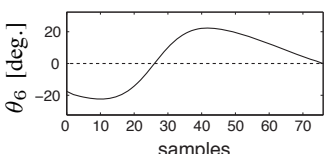

(b)

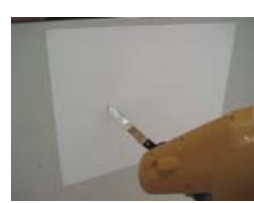

(c) $t=0 \mathrm{~s}$

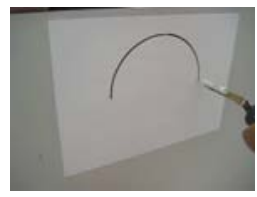

(e) $t=55 \mathrm{~s}$

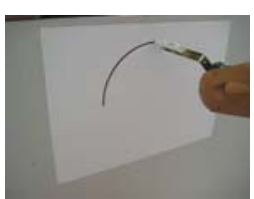

(d) $t=28 \mathrm{~s}$

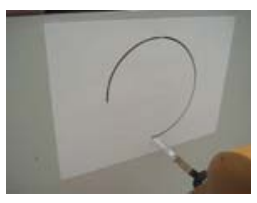

(f) $t=83 \mathrm{~s}$

Fig. 4. Example 1: (a) Trajectory of the end-effector; (b) Time history of the joint angles (solid); (c)-(f) Snapshots from the experiment.

\section{APPLICATIVE EXAMPLES}

This section presents two examples demonstrating the flexibility and ease of use of KCT in real scenarios. The first example shows an elementary application of the trajectory control functions (e.g., for painting, welding or assembling tasks). The second example reflects authors' personal interest in robot vision. In fact, the effectiveness of a camera rotation estimation method based on the geometry of planar catadioptric stereo (PCS) sensors [13] is tested using KCT.

The experimental results we will illustrate in the next subsections, have been obtained using the KUKA KR3 manipulator with KR C3 controller ${ }^{1}$.

\section{A. Drawing a circle}

Suppose we wish to draw the circle (in millimeters),

$x(k)=600, \quad y(k)=150 \cos (k), \quad z(k)=150 \sin (k)+310$,

$k \in[0,3 \pi / 2]$, on a paper board, with a pen mounted on the flange of the KUKA KR3 manipulator. To achieve this goal, we have first to initialize the robot using the command kctinit('KR3'). The TCP/IP communication between kctserver and $\mathrm{KCT}$ is then established with kctclient ('192.168.1.0'). The software bounds are set with the commands,

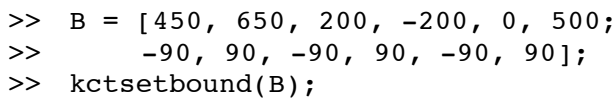

To draw the circle, we generated a matrix $\mathbf{P}$ of points using the following lines of code:

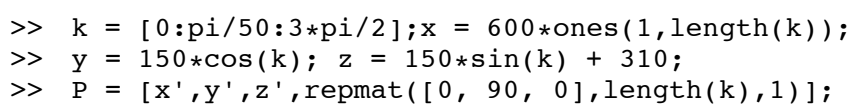

We finally called the function $\operatorname{kctpathxyz}(P, t p, 1)$, with time step $t p=1$. Fig. 4(a) shows the trajectory of the endeffector and Fig. 4(b) the time history of the joints angles, as returned by kctpathxyz setting to 1 its third argument. Figs. 4(c)-(f) show four snapshots of the real robot during the circular motion.

\footnotetext{
${ }^{1}$ The videos of the experiments are available at the web page:
} http://sirslab.dii.unisi.it/vision/kct

\section{B. Planar catadioptric stereo: camera rotation estimation}

Let us consider the planar catadioptric stereo (PCS) system [14] shown in Fig. 5(a). A pinhole camera mounted on the effector of the KUKA manipulator, observes a structured 3-D scene directly and reflected through two planar mirrors. Let us suppose we wish to estimate (using only the visual information), the rotation matrix $\mathbf{R}_{w}^{c}$ between the camera frame $\left\langle x_{c}, y_{c}, z_{c}\right\rangle$ and the mirrors' reference frame $\left\langle x_{w}, y_{w}, z_{w}\right\rangle$ while the camera moves with time (see Fig. 5(b)). By taking advantage of the epipolar geometry between the real camera and the virtual cameras associated to the two mirrors, a closed-form formula for $\mathbf{R}_{w}^{c}$ has been determined in [13, Prop. 8]. In order to test the robustness of this solution in a real-world setting, we moved the camera along a given trajectory and compared the estimated and actual roll-pitch-yaw angles of $\mathbf{R}_{w}^{c}$. Since we are interested in the camera's rotation, it is convenient to refer robot's motion with respect to the frame of the camera $\left\langle x_{c}, y_{c}, z_{c}\right\rangle$ in the initial point of the trajectory, i.e., for $t=0$. We then performed the following change of frame after the initialization/connection step:

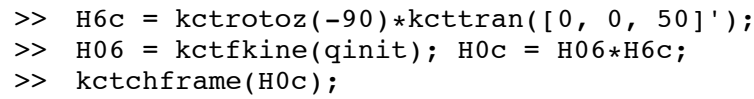

where $\mathbf{H}_{6}^{c}$ (known) and $\mathbf{H}_{0}^{6}$ are the homogeneous transformation matrices between the camera and the endeffector frames, and between the end-effector and the base frames, respectively. qinit is the vector of the joint angles of the robot in the initial point of the trajectory. kctdrivegui has been used to drive the eye-in-hand robot through other 14 points, where the real and mirrorreflected scenes were clearly visible. The value of the joint angles in the via points has been collected in the $15 \times 6$ matrix $\mathbf{Q}$ and the function [robotinfo, warn] $=\operatorname{kctpathjoint}(Q, t p, 0)$ with $t p=1$, has been called. The actual rotation matrices $\mathbf{R}_{w}^{c}$ along the trajectory have been obtained using the following commands (the rigid motion between $\left\langle x_{c}, y_{c}, z_{c}\right\rangle$ for $t=0$ and $\left\langle x_{w}, y_{w}, z_{w}\right\rangle$ has been measured in the real setup: it corresponds to a rotation of an angle $\alpha=90^{\circ}$ about the $x$-axis and to 


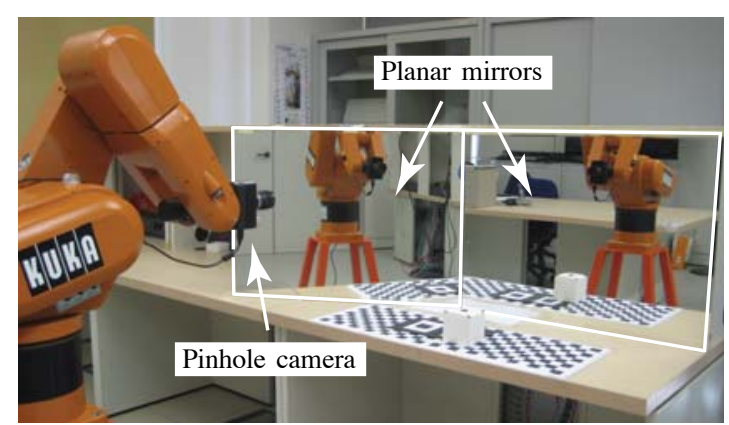

(a)

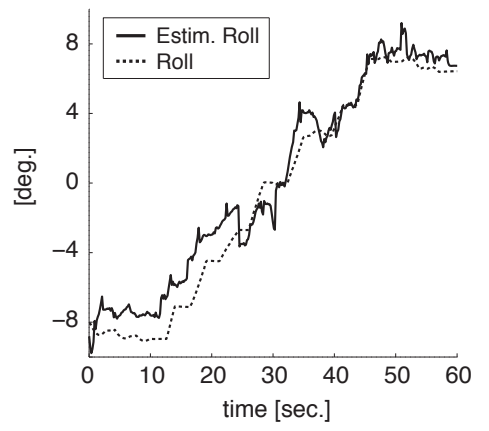

(c)

(d)

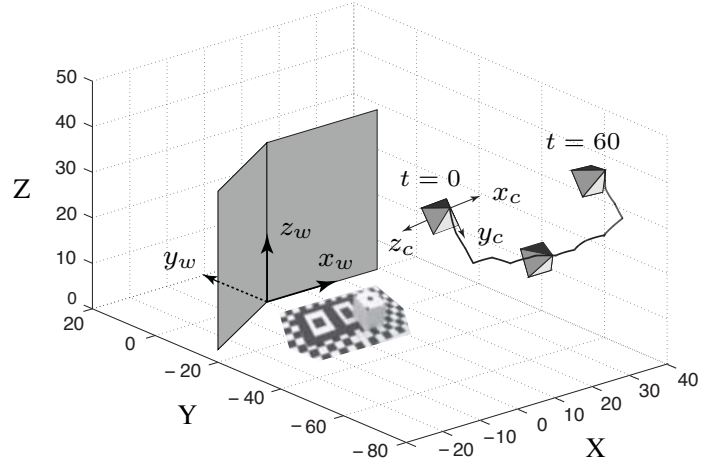

(b)

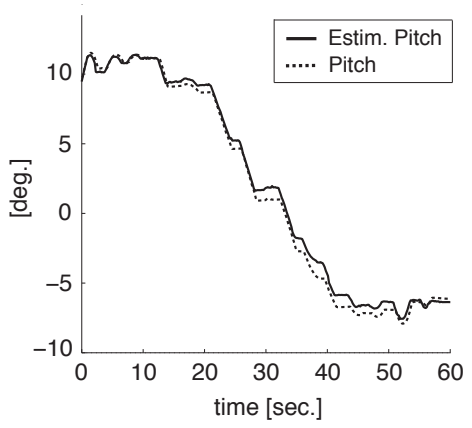

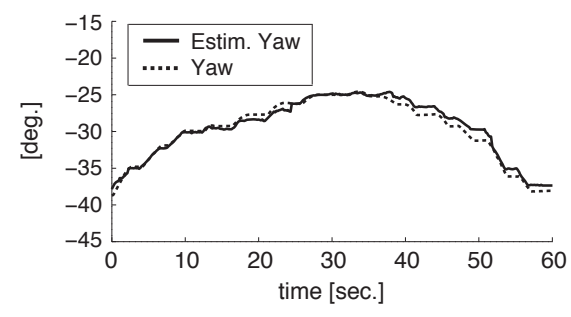

(e)

Fig. 5. Example 2: (a) PCS experimental setup; (b) 3-D trajectory of the camera with respect to the mirrors; (c)-(e) Time history of the estimated (solid) and actual (dash) roll-pitch-yaw angles of $\mathbf{R}_{w}^{c}$.

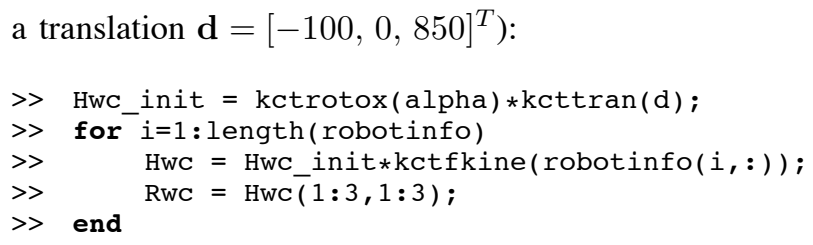

The time history of the roll-pitch-yaw angles of the matrices $\mathbf{R}_{w}^{c}$ thus computed (dash), together with the estimated values (solid), is shown in Figs. 5(c)-5(e).

\section{CONCLUSIONS AND FUTURE WORK}

This paper describes an open-source MATLAB toolbox for motion control of KUKA robot manipulators. The KUKA control toolbox $(\mathrm{KCT})$ runs on a remote computer connected with the KUKA controller via TCP/IP, and it includes a heterogenous set of functions for kinematics computation, trajectory planning and graphical display. The flexibility and reliability of the toolbox has been demonstrated via two real-word examples. KCT is an ongoing software project: work is in progress to extend the compatibility of the toolbox to all (not necessarily 6 DOF) small and low payload KUKA robots. We also aim to create a robot simulator for off-line validation of motion control tasks and extend the functionality of KCT to the Simulink environment.

\section{REFERENCES}

[1] MATLAB and Simulink for Technical Computing. The MathWorks Inc., USA. [Online]: http: //www. mathworks.com/.

[2] P.I. Corke. A Robotics Toolbox for MATLAB. IEEE Rob. Autom. Mag., 3(1):24-32, 1996.
[3] K. Yoshida. The SpaceDyn: a MATLAB Toolbox for Space and Mobile Robots. In Proc. IEEE/RSJ Int. Conf. Intel. Robots Syst, pages 1633-1638, 1999.

[4] A. Breijs, B. Klaassens, and R. Babuška. Automated design environment for serial industrial manipulators. Ind. Robot, 32(1):32-34, 2005.

[5] G.L. Mariottini and D. Prattichizzo. EGT for Multiple View Geometry and Visual Servoing: Robotics and Vision with Pinhole and Panoramic Cameras. IEEE Robot. Autom. Mag., 12(4):26-39, 2005.

[6] P.I. Corke. The Machine Vision Toolbox: a MATLAB toolbox for vision and vision-based control. IEEE Robot. Autom. Mag., 12(4):16$25,2005$.

[7] R. Falconi and C. Melchiorri. RobotiCad: an Educational Tool for Robotics. In Proc. 17th IFAC World Cong., pages 9111-9116, 2008.

[8] W.E. Dixon, D. Moses, I.D. Walker, and D.M. Dawson. A SimulinkBased Robotic Toolkit for Simulation and Control of the PUMA 560 Robot Manipulator. In Proc. IEEE/RSJ Int. Conf. Intel. Robots Syst, pages 2202-2207, 2001.

[9] M. Casini, F. Chinello, D. Prattichizzo, and A. Vicino. RACT: a Remote Lab for Robotics Experiments. In Proc. 17th IFAC World Cong., pages 8153-8158, 2008.

[10] KUKA Robotics Corporation [Online]: http: //www . kuka-robotics.com/.

[11] G. Maletzki, T. Pawletta, S. Pawletta, and B. Lampe. A ModelBased Robot Programming Approach in the MATLAB-Simulink Environment. In Int. Conf. Manuf. Res., pages 377-382, 2006. [Online]. http://www.mb.hs-wismar.de/ gunnar/ software/KukaKRLTbx.html.

[12] M.W. Spong, S. Hutchinson, and M. Vidyasagar. Robot Modeling and Control. Wiley, 2006.

[13] G.L. Mariottini, S. Scheggi, F. Morbidi, and D. Prattichizzo. Planar Catadioptric Stereo: Single and Multi-View Geometry for Calibration and Localization. In Proc. IEEE Int. Conf. Robot. Automat, pages 1510-1515, 2009.

[14] J. Gluckman and S.K. Nayar. Catadioptric Stereo using Planar Mirrors. Int. J. Comput. Vision, 44(1):65-79, 2001. 